\title{
A MAC Protocol for Provisioning QoS and Energy-Efficiency in WSNs
}

\author{
Zahra Zarei, S. Mostafa Safavi, and Akbar Abbasi
}

\begin{abstract}
Reliable environment monitoring and information collection with an energy efficiency mechanism is a goal in new wireless sensor network applications. In some applications, reliability and delay are two important and vital parameters. Lifetime improvement in wireless sensor networks is also a challenging issue. Therefore, in this paper, a new protocol is proposed for solving these problems. The proposed protocol uses CSMA/CA basic algorithm and some simple mechanisms to improve energy-efficiency and network reliability with reasonable end-to-end delay. In this paper the simulation results of the proposed protocol are compared with both S-MAC and 802.11 protocols in the same condition that show better performance.
\end{abstract}

Index Terms-Energy efficiency, MAC protocols, quality of service (QoS) provisioning, reliability, wireless sensor network.

\section{INTRODUCTION}

Wireless sensor networks (WSNs) have been proposed for monitoring of physical phenomena with little or no human attendance. Inherent to such concept is one of the basic assumptions that every node in a WSN is equipped with a battery, and it has usually been assumed that the battery is irreplaceable [1]. Research in WSN has focused on providing energy-efficient operation of each node that provides as long lifetime of WSN as possible. However, recent advances in research on sensing technologies, e.g. sensor cameras [2], multimedia applications in sensor networks, e.g. target tracking in battlefield for military application, and various mission critical applications, e.g. in networked control systems [3] have introduced delay or packet-loss sensitive traffic in wireless sensor networks.

Since sensor networks are generally deployed in inhospitable terrains and consist of a dense deployment of sensor nodes, furthermore, wireless communication is inherently unpredictable and error-prone; hence, it becomes essential to employ a MAC protocol that efficiently shares the wireless channel. For many real-life applications of sensor networks, guaranteed delivery of data is very important. A critical event detected by the sensor network should be delivered to the user as soon as possible. Thus, for sensor networks, reliability and latency are also important design parameters [4]. However, high reliability and low latency come at the cost of more energy expenditure. Here assumed the criteria such as source to sink delay, reliability or

Manuscript received April 9, 2012; revised July 1, 2012.

Z. Zarei is with Department of Electrical Engineering, Malek Ashtar University of Technology, Tehran, Iran (e-mail: z.zarei0631@yahoo.com).

S. M. Safavi is with Amir Kabir University of Technology, Tehran, Iran (e-mail: msafavi@aut.ac.ir).

A. Abbasi is with Department of Information Technology Douran Company, Tehran, Iran (e-mail: aabbasi@douran.com). acceptable data loss rate in wireless sensor networks are usually referred as quality-of-service. In this paper, we present a novel Medium Access Control (MAC) protocol that ensures reliability and low latency in contention-based channel conditions while it focuses on minimizing energy consumption.

The rest of the paper is organized as follows. Sections II, III, IV discuss the proposed MAC protocol, Energy-Aware QoS Provisioning Protocol (EAQPP). Section V presents the performance evaluation of comparing the proposed protocol with two famous protocols S-MAC and IEEE 802.11. Section VI concludes the paper and highlighting its contributions.

\section{The Proposed Protocol: Assumptions}

In the design of the proposed protocol, two issues are considered: providing improved QoS and lower energy consumption compared to previous related papers. The proposed Energy-Aware QoS Provisioning Protocol (EAQPP) is a development of CSMA/CA protocols [5] and RMAC [4] in MAC layer and is similar with TinyOS MAC protocol [6]. Therefore, EAQPP has two main features: carrier sense and using of back-off algorithm. In other words, in the proposed protocol, virtual carrier sense mechanism (RTS/CTS) [7] is not used but sensors use back-off algorithm at time of occupation of communication channel. In EAQPP protocol, we focus on two parameters of QoS: reliability and delay, and for avoidance of sleep delay, we utilize wakeup on-demand technique [8].

In EAQPP protocol, we have some reasonable assumptions [4]:

Each node has one ID and knows its neighbor IDs.

The network topology is static, i.e., the sensor nodes are static and the only possible change in the network topology is due to discharge of sensor nodes.

Layer 3 protocols are responsible of building routing tables in order to send packets to their destinations.

If some duplicated packets are transmitted, with assumption of using aggregation mechanism, they are dropped.

To achieving improved reliability, we assume that Bit Error Rate (BER) is specific and with this parameter, we can obtain packet error rate.

$$
P_{e}=1-(1-e)^{S}
$$

e: BER

s: Number of bits in packet

It is assumed that each node have a Data Radio (DR) and also a Wakeup Radio (WR) [9], [10], [11]. 


\section{The PROPOSED PROTOCOL: BASIC FUnCTIONS}

In the proposed protocol (EAQPP), energy efficiency is implemented with using transceiver's sleep and wakeup mode. For having QoS in data link layer, some mechanism is implemented: Using Positive Acknowledgement mechanism in an implicit manner and also, sending negative acknowledgement packet (NACK) [12]. The general functions of the proposed protocol are as following:

The total nodes are initially in sleep mode, i.e., DR is in sleep mode but WR is in listen mode.

When a node wants to send a packet, it sends a wakeup tone after a random back-off duration, and thus after detection of wakeup tone by all neighbor nodes, they switch on their DRs.

The sender node turns on its DR and propagates a filter packet [6]. This packet contains receiver's ID, to identify the intended node.

When the intended node receives the filter packet, turns on its DR, but other nodes turn off their DRs and go to sleep mode.

The sender transmits its packet to next node via DR and then goes to sleep mode. The general functions of the source node are shown in following section.

Until receiving the complete packet, the receiver keeps its DR turn on. If the packet is successfully received, the terminal receiver go to sleep by sending ACK packet (this operation is only done at terminal receiver and intermediate nodes do not send ACK packet. The intermediate nodes only use implicit acknowledgement mechanism). Otherwise, if the packet is not successfully received, the receiver drops it and sends NACK packet, then it goes to sleep.

It is considered that before sending wakeup tone via WR, the source node does a random backoff. The following formula calculates the random backoff:

$$
\text { Random_Backoff }=\text { Uniform }\left[0,2^{C W}\right] \times 2 \tau
$$

During this time, if the channel is busy, the sensor node stops its counter and waits till the channel is idle. At the end, the sensor node listens to channel, if the channel is busy, it again calculates Contention Window $(\mathrm{CW})$ and goes to sleep mode, then after a random back-off, sends a wakeup tone. Thereafter the sender listens to primary channel, before sending packet filter. If the primary channel is busy, it goes to sleep and repeats the process by sending wakeup signal after a random back-off. It is probable that after wakeup, the receiver can not receive filter packet. Two reasons can cause this problem. One may happen when the sender node finds the primary channel busy and is unable to send the filter packet; second reason is due to packet collision. To handle such situations, after turning on DR, a node waits for the time duration required to send a filter packet. If it doesn't receive any filter packet within this duration, it goes back to sleep mode. The functions of Source node, intermediate node and end (destination) node have been shown in Figs. 2, 3, 4.

\section{The Proposed Protocol: Specific FeAtures}

The proposed protocol has some features to significantly improve QoS and energy efficiency as follows:

- When an intermediate node receives a data packet successfully, if the channel is idle, it skips backoff and transmits the packet to the next node immediately. Utilizing this method reduce the total network delay. To do so, when the intermediate node receives a data packet successfully, it sends a wakeup tone and then sends a filter packet via WR. After sending filter packet, it sends data packet via DR. Sending of wakeup tone by intermediate node, with regard to broadcast propagation property in wireless environment, can considered as an implicit ACK for the previous node. For example, in Fig. 1, node A sends a packet to node B. If node $B$ receives the packet correctly, it skips back-off and sends a wakeup tone to initiate transmission with node $\mathrm{C}$. Therefore, after a delay equals to the required time for processing, propagation and switching between modes, node A receives this wakeup tone and considers it as a positive ACK. If node A doesn't receive implicit ACK, after backoff duration it sends again a wakeup tone and a filter packet to retransmit the packet to node B. Finally, if the end node (node D) receives the packet correctly, it sends an explicit ACK to the previous node; otherwise it sends a NACK packet to the previous node.

To safely implement skip-backoff mechanism, we must set the minimum $\mathrm{CW}_{\min }$ such that average random back-off is greater than processing delay.

$$
A I B=2^{C W_{\text {min }-1}} \times 2 \tau>T_{\operatorname{Pr} o c}
$$

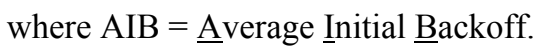

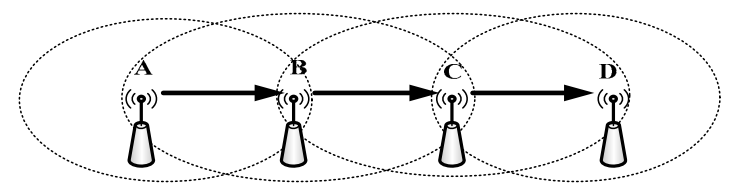

Fig. 1. Relation of nodes.

In the proposed protocol, instead of fixing the number of retransmission attempts for any packet, based on channel state, the number will be changed adaptively. In a situation with high channel error many packets may be corrupted at physical layer. Therefore, the number of retransmission attempts, with regard to information related to channel BER that is obtained by previous packets, is increased for achieving network reliability. If $\mathrm{P}_{\mathrm{e}}$ is packet error rate, then the number of retransmission attempts is calculated as follows:

$$
R_{-} A=T_{\text {Attempt }}+\left[1 /\left(1-P_{e}\right)\right]
$$

where $\mathrm{T}_{\text {Attempt }}$ is default number for transmission attempts.

In each node for optimum usage of resources, buffer management operation is done. This protocol each packet in addition to common fields included the fields of TTL, Birth_Time_Stamp (BTS), and Current_Time_Stamp (CTS). TTL shows the number of remaining nodes to final destination and its value decreases in each node. This field is created to discard wandering packets. The TTL value is set by the source of the packet. This operation in wireless sensor networks is done by utilizing routing protocols and GPS mechanisms. The BTS is instantaneous timestamp when the packet had been initially generated. Initially, BTS value equals to CTS, and they are set by source node. Each intermediate node adds to the CTS value the duration time 
that a packet waits in queue. In other words, each intermediate node adds a Communication Duration (CD) to this field. One Communication Duration is the time taken by the packet to transmit over one hop. For each node, According to the values of these three parameters, the following comparison is done:

$$
P L T+B T S>\left\{\begin{array}{l}
C T S+T T L \times C D \\
C T S
\end{array}\right.
$$

where PLT (Packet Life Time) is the maximum waiting time for a packet.

This parameter depends on maximum buffer length and packet processing rate. If for each packet one of the above conditions is true then the packet is dropped. With this operation, buffer related to each node is freed for arriving packets and caused to reduce end-to-end latency of the system. Meanwhile, this method predicts whether lifetime of a packet will be expired before reaching to destination, and then drops aging packets that causes total energy-saving in network.

- When an implicit ACK is received by the sender node, it sets a timer (ReceiveTimer) for time equal to one communication period. During this period (and until it is expired), the sender node drops the received wake-up signals (or it can turn off its WR radio). This is done because during this interval its next hop node is engaged in forwarding the packet and this node would not be able to receive packets without collision. Utilizing RecieveTimer prevents unnecessary energy consumption and makes energy-saving in the wireless sensor network, since the node can sleep during this period and prevents it from receiving the collided packets.

- As mentioned earlier, when a node have a packet to send, it sends a wakeup signal to wakeup all its neighbors. After that it sends a filter packet to specify its intended receiver. After processing of the filter packet the other neighbors go to sleep mode. They also refrain from sending or receiving of packets during a period of $2 *$ Communication Duration. To do this, both CW and ReceiveTimer is set for a period of $2 *$ Communication Duration. This caused to prevent the neighbors of the sender and receiver node from overhearing and unnecessary stat changes, during the required time for sending a packet from sender to receiver and from the receiver node to its next hop node. This prevents possible collisions due to hidden node interference.

\section{Simulation Results}

NS-2 simulator is used to simulate and analyze of the proposed protocol. To effectively show the benefits of the proposed protocol, it is compared to S-MAC [13], [14] and basic 802.11 protocols. The evaluation metrics in the simulation are as follows:

Packet Delivery Ratio (PDR): This is the ratio of the total number of packets successfully received at the destination to the total number of transmitted packets by the source nodes over the simulation time. This parameter can be expressed as the measure of system reliability.

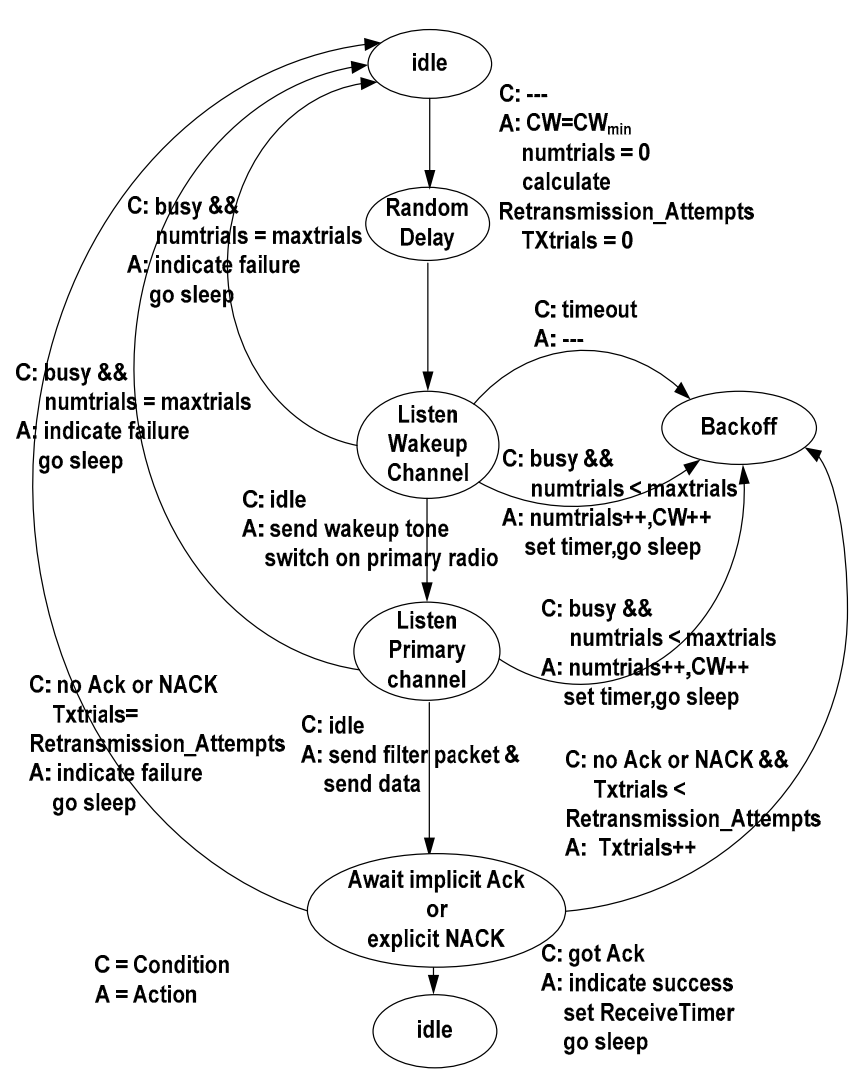

Fig. 2. Source node functions.

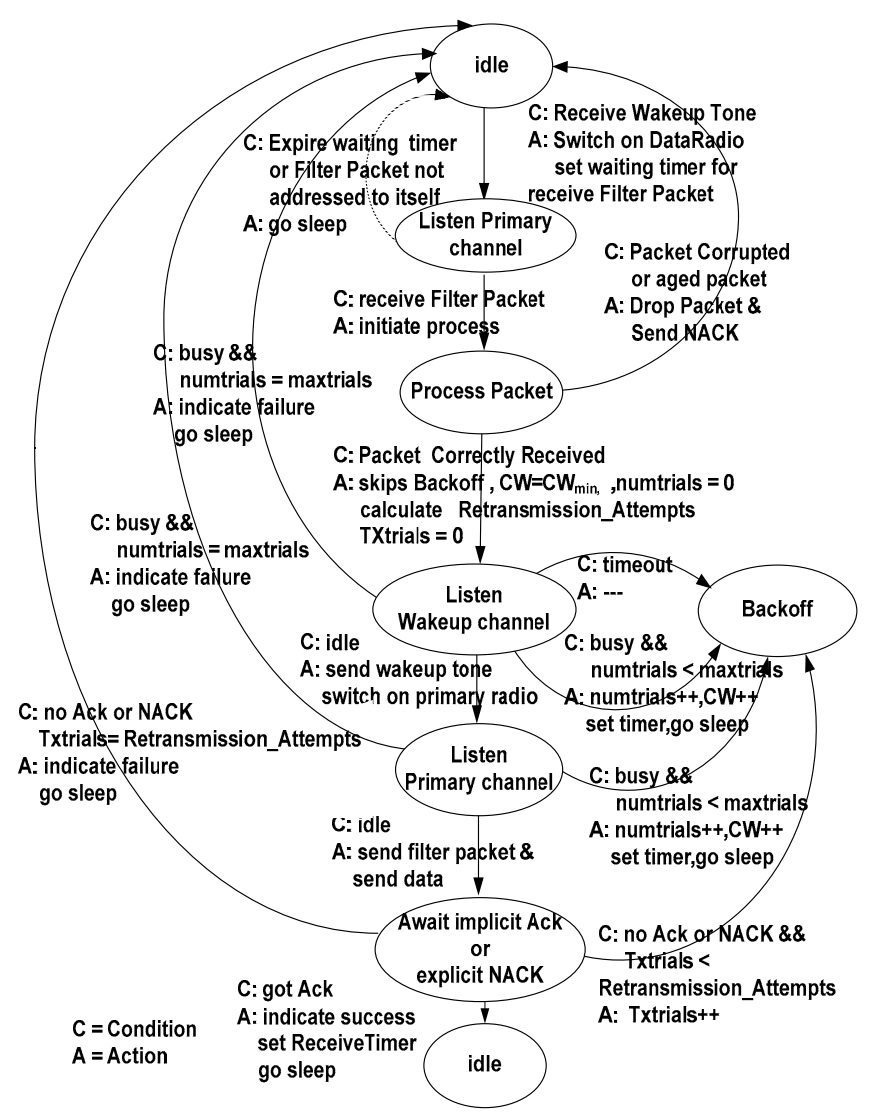

Fig. 3. Intermediate node functions. 


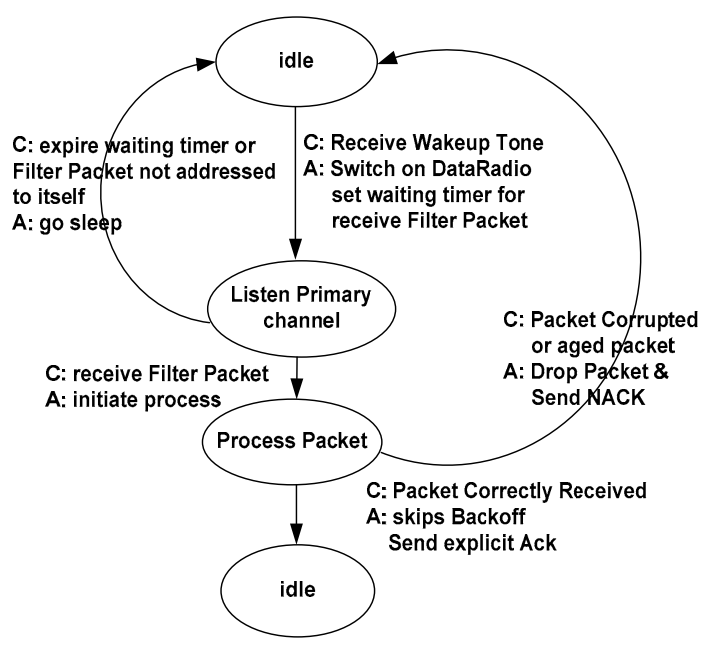

Fig. 4. End node functions.

Delay per Node: This is the time average time between the generation of a packet and successfully receiving of the packet at the destination. In the other words, this parameter indicates average of packet delay in each node on its path to the destination.

Energy Consumption per Node: This is the average energy expended by a sensor node for each successful reception at the destination.

Some required simulation parameters have been listed in Table I. In this Table, Radio parameters are taken from Telos specifications [15] and Chipcon CC2420 [16] and packet formats are taken from 802.15.4 Standard [17]. Some descriptions about this table are as follows:

$P_{T x_{-} \text {on }} / P_{T x_{-} \text {off: }}:$ The necessary power for turning on/off the primary radio.

$T_{T x_{-} \text {on }}$ and $T_{T x_{\_} \text {off: }}$ The necessary time for switching between wakeup and sleep modes.

$P_{\text {wakeup_Tx }} / P_{\text {wakeup_Rx }}$ : The necessary power for transmitting/receiving wakeup tone.

For simulation of the proposed protocol, we assumed that 100 nodes are uniformly distributed in $1^{\mathrm{km}} \times 1^{\mathrm{km}}$ sensor field and transmission range for each node is 100 meters. Each simulation is run for 100 seconds and is averaged over 5 iterations. Packet generation, at source node, is based on Poisson process and packet generation rate can change between $1 \mathrm{pkts} / \mathrm{sec}$ to $10 \mathrm{pkts} / \mathrm{sec}$. To measure the value of the protocol reliability, different error rate is assigned to communication channel, from 0 to 0.4 with step of 0.2 . This error rate means that the probability with which a successfully received packet will be dropped by the receiver considering the packet as corrupted due to channel error. Energy consumption model considered for this simulation is similar to basic model of NS-2. In the simulation, the total intermediate node from packet source to its destination is 10 .

TABLE I: SIMULATION PARAMETERS.

\begin{tabular}{ll}
\hline \multicolumn{1}{c}{ Parameter } & \multicolumn{1}{c}{ Value } \\
\hline Packet size & 77 bytes \\
Filter size & 14 bytes \\
Ack size & 9 bytes \\
slotTime & $100 \mu \mathrm{s}$ \\
$\mathrm{T}_{\mathrm{SIFE}}$ & $20 \mu \mathrm{s}$ \\
$\mathrm{CW}_{\min }$ & 1 \\
$\mathrm{CW}_{\max }$ & 4 \\
$\mathrm{Tx}_{-} \mathrm{Attempts}$ & 5 \\
$\mathrm{P}_{\mathrm{Tx}}=\mathrm{P}_{\mathrm{Rx}}$ & $41 \mathrm{~mW}$ \\
\hline
\end{tabular}

\begin{tabular}{ll}
\hline $\mathrm{P}_{\text {sleep }}$ & $0.015 \mathrm{~mW}$ \\
$\mathrm{P}_{\text {Tx_on }}=\mathrm{P}_{\text {Tx off }}$ & $35 \mathrm{~mW}$ \\
$\mathrm{~T}_{\text {Tx on }}$ & $580 \mu \mathrm{s}$ \\
$\mathrm{T}_{\text {Tx_off }}=\mathrm{P}_{\text {wakeup Rx }}$ & $50 \mu \mathrm{s}$ \\
$\mathrm{P}_{\text {wakeup Tx }}$ & $0.015 \mathrm{~mW}$ \\
Buffer size & 30 packets \\
Packet lifetime & $0.3 \mathrm{msec}$ \\
TTL & 30 \\
Tproc & $10 \mu \mathrm{s}$ \\
Modulation & BPSK \\
Center Ferequency & $916.50 \mathrm{MHz}$ \\
B.W & $400 \mathrm{kHz}$ \\
Max. Data Rate & $40 \mathrm{kbps}$ \\
\hline
\end{tabular}

The simulation results are shown in Fig. 5, Fig.6, and Fig. 7. The figures show that when the channel is error-free, PDR values for all three protocols are identical, but the proposed protocol has lower average delay per node, as compared to the other protocols (maximum of $15 \%$ less than S-MAC and $8 \%$ less than 802.11). Also, in general the energy consumption average of the proposed protocol is less than two other protocols ( $97 \%$ less than 802.11 and $49 \%$ less than S-MAC protocol).

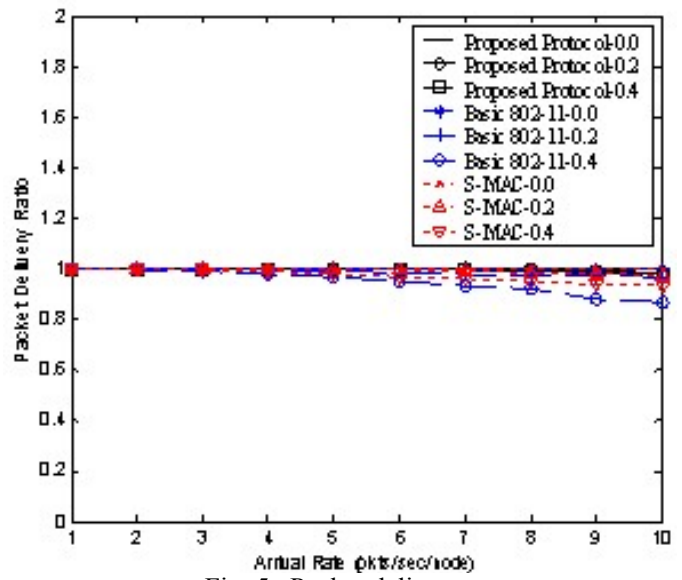

Fig. 5. Packet delivery rate.
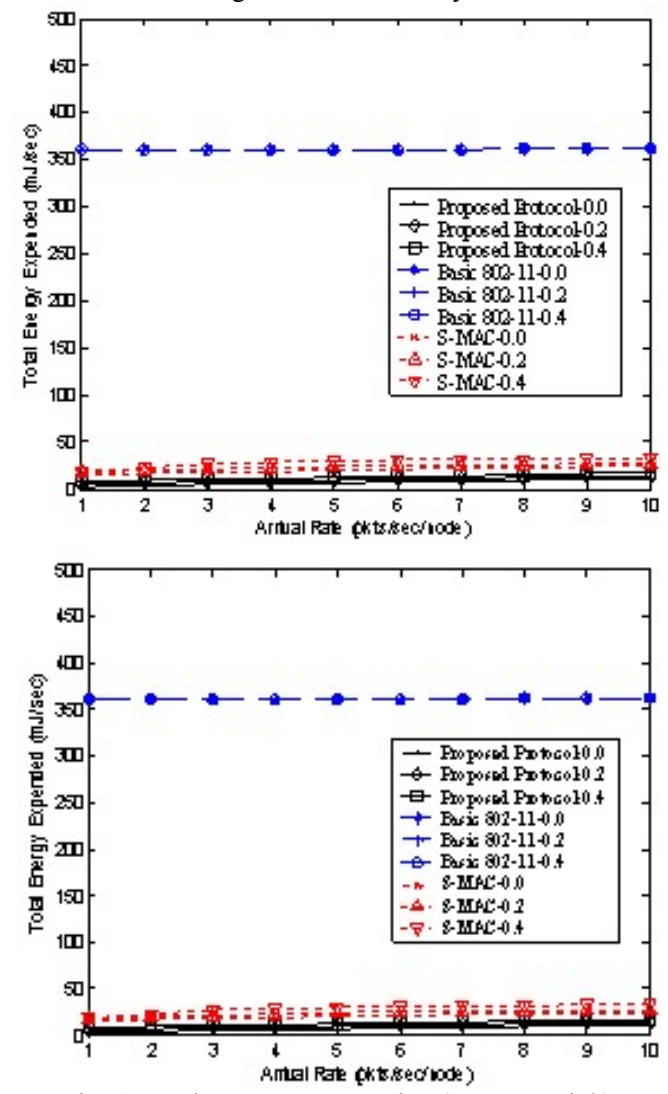

Fig. 6. Total energy consumption (source to sink). 


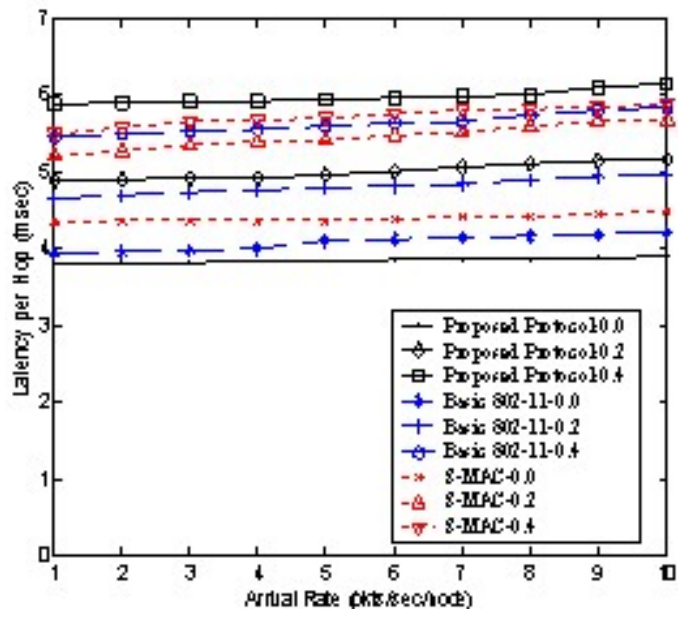

Fig. 7. Delay per node.

\section{CONCLUSIONS}

The simulation results of the three protocols in different situations of channel error rate and packet transmission rate show that the proposed protocol has superior performance, better PDR and energy-efficiency with acceptable delay compared to the other protocols. The results show also high scalability to increase the packet generation rate. Then the proposed protocol in data link layer has acceptable performance for applications that require high reliability and lower delay.

\section{REFERENCES}

[1] N. Zogović, G. Dimić, and D. Bajic, "Wireless Sensor Networks: QoS Provisioning at MAC and Physical Layers," in Proceeding of 17th Telecommunications forum TELFOR 2009, Serbia, November, 2009.

[2] I. F. Akyildiz, T. Melodia, and K. R. Chowdury, "Wireless multimedia sensor networks: a survey," IEEE Wireless Communications, vol.14, issue 6, pp. 32-39, December 2007.

[3] J. Baillieul and P. Antsaklis, "Control and communication challenges in networked real-time systems," in Proceedings of IEEE, vol. 95, Issue: 1, January 2007.
[4] R. Biswas, V. Jain, C. Ghosh, and D. P. Agrawal, "On-Demand Reliable Medium Access in Sensor Networks," in Proceedings of IEEE International Symposium on a World of Wireless, Mobile and Multimedia Networks (WoWMoM), Niagara-Falls, Buffalo, June 2006.

[5] L. Kleinrock and F. A. Tobagi, "Packet Switching in Radio Channels: Part 1 Carrier Sense Multiple Accaesss Models and their Throughput-/Delay-Characteristic," IEEE Transactions on Communications, vol. 23, no. 12, pp. 1400-1416, 1975.

[6] A. Woo and D. E. Culler, "A Transmission Control Scheme for Media Access in Sensor Networks," in Proceedings of the 7th Annual ACM International Conference on Mobile Computing and Networking (MobiCom), Rome, Italy, pp. 112-117, 2001.

[7] IEEE Standard for Wireless LAN Medium Access Control (MAC) and Physical Layer (PHY) Specifications, The Editors of IEEE 802.11, November 1997.

[8] L. C. Zhong, R. C. Shah, C. Guo, and J. M. Rabaey, "An Ultra-Low Power and Distributed Access Protocol for Broadband Wireless Sensor Networks," in IEEE Broadband Wireless Summit, Las Vegas, NY, May 2001.

[9] M. J. Miller and N. H. Vaidya, "Minimizing Energy Consumption in Sensor Network using a Wakeup Radio," in Proceedings of IEEE WCNC 2004, Atlanta, Georgia, March, 2004.

[10] C. Schurgers, V. Tsiatsis, S. Ganeriwal, and M. Srivastava, "Optimizing Sensor Networks in the Energy-Latency-Density Space," IEEE Transactions on Mobile Computing, vol. 1, no. 1, pp. 70-80, January-March 2002.

[11] M. J. Miller and N. H. Vaidya, "A MAC Protocol to Reduce Sensor Network Energy Consumption Using a Wakeup Radio," IEEE Transactions on Mobile Computing, vol. 4, no. 3, pp. 228-242, May-June 2005.

[12] S.-J. Park and R. Sivakumar. "Poster: Sink-to-Sensors Reliability in Sensor Networks," in Proceedings of the 4th ACM International Symposium on Mobile Ad Hoc Networking and Computing (MOBIHOC), Annapolis, MD, June 2003.

[13] W. Ye, J. Heidemann, and D. Estrin, "An Energy-Efficient MAC Protocol for Wireless Sensor Networks," in Proceedings of INFOCOM 2002. IEEE Press, New York, June 2002.

[14] W. Ye, J. Heidemann, and D. Estrin, "Medium Access Control with Coordinated, Adaptive Sleeping for Wireless Sensor Networks," IEEE/ACM Transactions on Networking, 2004.

[15] J. Polastre, R. Szewczyk, and D. Culler, "Telos: Enabling Ultra-Low Power Wireless Research," in Proceedings of the 4th International Conference on Information Processing in Sensor Networks: Special track on Platform Tools and Design Methods for Network Embedded Sensors (IPSN/SPOTS), Los Angele, California, pp. 86-92, 2005.

[16] Chipcon SmartRF® CC2420 Datasheet (rev 1.3). (2005-10-03). [Online]. Available: http://www.chipcon.com

[17] IEEE 802.15.4 - 2003: Wireless MAC and PHY spec. for Low Rate Wireless Personal Area Networks. 